\title{
Publisher Correction: On our bookshelf
}

Marios Karouzos

Correction to: Nature Astronomy https://doi.org/10.1038/s41550-017-0316-9, published online 2 November 2017.

In the version of this Books and Arts originally published, the book title Spectroscopy for Amateur Astronomy was incorrect; it should have read Spectroscopy for Amateur Astronomers. This has now been corrected. 